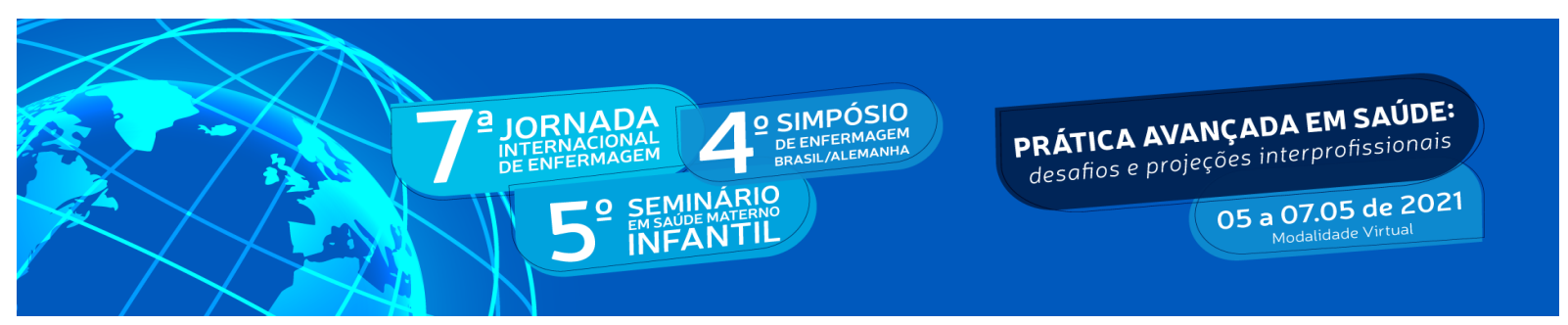

DOI: http://doi.org/10.48195/jie2021-014

\title{
O USO DOS ANTIBIÓTICOS NA REMOÇÃO DOS TERCEIROS MOLARES: REVISÃO DE LITERATURA. Caroline Bortolas de Carvalho' ${ }^{1}$, Jovito Skupien ${ }^{2}$
}

\section{RESUMO}

A remoção dos terceiros molares é uma das cirurgias mais frequentes na odontologia. De uma maneira geral, após o procedimento é muito comum a prescrição de antibióticos por parte dos cirurgiões-dentistas com o intuito de prevenir complicações pós operatória como a sintomatologia dolorosa, infecção, trismo e alveolite. Uma busca na literatura foi realizada utilizando as bases de dados PubMed e Cochrane com o objetivo de avaliar as evidências científicas que justifiquem a prescrição de antibióticos para pacientes saudáveis, submetidos a exodontia de terceiros molares. Deve-se avaliar bem a indicação de antibióticos para prevenção de infecções, levando em consideração alguns fatores que podem aumentar o risco de complicações infecciosa. Ainda, o uso da profilaxia antibiótica não se mostrou eficaz para redução das complicações infecciosas, devendo ser utilizado somente em pacientes imunocomprometidos com risco de endocardite bacteriana.

Palavras-chave: Antibioticoprofilaxia; Cirurgia bucal; Dente serotino.

\begin{abstract}
The removal of third molars is one of the most frequent surgeries in dentistry. In general, after the procedure, it is very common for dentists to prescribe antibiotics in order to prevent postoperative complications such as painful symptoms, infection, trismus and alveolitis. A literature search was performed using PubMed and Cochrane databases in order to evaluate the scientific evidence that justifies the prescription of antibiotics for healthy patients, which were submitted to an extraction. The indication for antibiotics to prevent infections should be well evaluated, taking into account some factors that may increase the risk of infectious complications. In addition, the use of antibiotic prophylaxis has not been shown to be effective in reducing infectious complications, and should only be used in immunocompromised patients at risk for bacterial endocarditis.
\end{abstract}

Keywords: Antibiotic prophylaxis; Oral surgery; Serotine tooth.

\footnotetext{
${ }^{1}$ Caroline Bortolas de Carvalho/ - UFN - Carolbortolas@yahoo.com.br.

2 Jovito Skupien - UFN - skupien.ja@gmail.com
} 


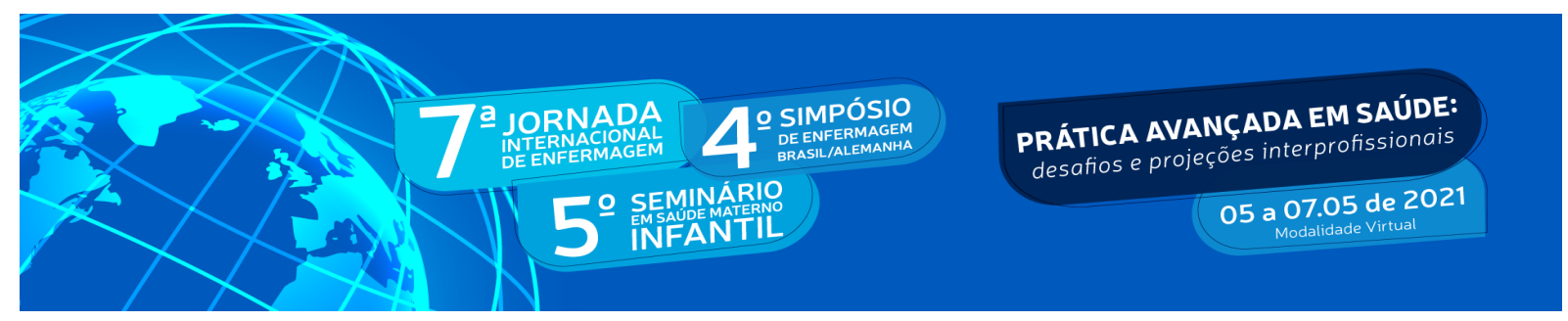

\section{INTRODUÇÃO}

A cirurgia para remoção dos terceiros molares é o procedimento muito comum na prática odontológica tendo sua etiologia principalmente relacionada a doença cárie, periodontal ou ainda por problemas inflamatórios relacionados a sua impactação (PHAM; NGUYEN, 2019; HAAG et al., 2017). Assim, quando necessário, a remoção dos terceiros molares pode resultar em uma série de complicações, incluindo dor, trismo, edema, sangramento, alveolite e infecções. As infecções relativas após a exodontia dos terceiros molares parece ter uma prevalência maior quando comparada aos demais dentes (AL-SHAMIRI, SHAWKY E HASSANEIN,2017; BRAIMAH et al., 2017; RALDI et al., 2019). A experiência do cirurgião, o tempo de cirurgia, pericoronarite e doenças debilitantes são considerados predisponentes para infecção pós operatória, em contrapartida, a técnica asséptica, hemostasia, o manejo delicado dos tecidos e a irrigação do sitio cirúrgico podem contribuir para diminuir os índices de infecção.( AL-SHAMIRI, SHAWKY E HASSANEIN, 2017; LODI et al., 2021; )

Mesmo com todos os cuidados necessários, alguns cirurgiões-dentistas, com a finalidade de reduzir a incidência de infecção pós-operatória, administram antimicrobianos ora de forma sistêmica, ora de forma profilática, constituindo uma prática muito comum no atual cenário da cirurgia dento alveolar (BRAIMAH et al., 2017; MARCUSSEN et al., 2016). O papel dos antibióticos de uso sistêmico para prevenção de infecção nas exodontias de terceiros molares ainda é muito questionado, diferentemente dos casos com pacientes suscetíveis a infecções tais como diabéticos, usuários crônicos de corticoides e aqueles com maior risco à endocardite, onde a profilaxia antibiótica já é algo consolidado na literatura científica (LODI et al., 2021).

Embora existam diversos protocolos na literatura, não há uma padronização ideal do uso ou não de medicamentos no pré e/ou pós-operatório, deixando a critério do cirurgião a escolha do melhor protocolo medicamentoso a ser realizado, que pode estar baseado de acordo com a sua formação acadêmica ou o seu próprio "know-how". 


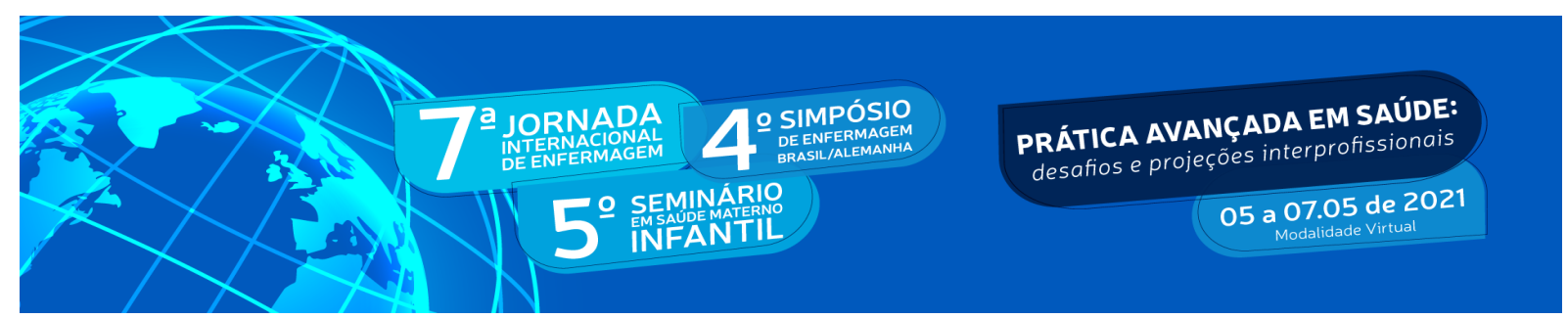

2. OBJETIVO

Avaliar as evidências científicas que justifiquem a prescrição de antibióticos para pacientes saudáveis, submetidos a extrações de terceiros molares.

\section{METODOLOGIA}

Trata-se de uma revisão de literatura a respeito dos antibióticos administrados de forma profilática (no pré-operatório), somente no pós-operatório, de forma contínua por 5 ou 7 dias, ou ainda de maneira conjunta, no pré e pós-operatório das cirurgias de terceiros molares e que investigaram a influência desta terapia medicamentosa nos sintomas de dor, trismo, edema e qualidade de vida no pós-operatório dos indivíduos frente a um procedimento cirúrgico.

A busca por artigos ocorreu através do auxílio das bases de dados online PubMed e Cochrane, utilizando as palavras-chaves ((third molar) and (extraction)) and ((complication)). Foram totalizados 478 artigos. Os critérios de inclusão foram artigos que abordassem a temática na área da saúde, com alto nível de evidência científica: ensaios clínicos randomizados, revisões sistemáticas e estudos epidemiológicos bem delineados e que observassem os itens citados previamente (prescrição de medicamento e desfechos). Como critério de exclusão utilizou-se o tempo de publicação, sendo, aqueles com o período maior de 10 anos foram excluídos. A busca e leitura dos artigos ocorreram de março de 2020 a março de 2021, sendo selecionados 24 artigos para a revisão de literatura.

\section{RESULTADOS E DISCUSSÃO}

Os terceiros molares são os dentes que geralmente erupcionam na cavidade bucal entre os 17 e 25 anos de idade podendo surgir em posições anômalas, com variações quanto a sua forma, tamanho e raízes, e, frequentemente, é o grupo dentário com maior probabilidade de retenção, pois são os últimos dentes a erupcionar na cavidade bucal (BRUNELLO et al.,2017; FERNANDES; ARMOND; FALCI, 2019; CAYMAZ; UYANIK, 2019; MAHAT et al., 2020).

Em alguns casos, a extração destes dentes se faz necessária. A remoção dos terceiros molares é uma das cirurgias mais frequentes realizadas pelos cirurgiões-dentistas (CDs) e especialistas em cirurgia bucomaxilofacial a nível ambulatorial podendo estar associado ao aparecimento de diversas alterações relacionadas ao procedimento tais como: sintomatologia dolorosa, edema, equimose, trismo e comprometimento de estruturas importantes (MAJID, 


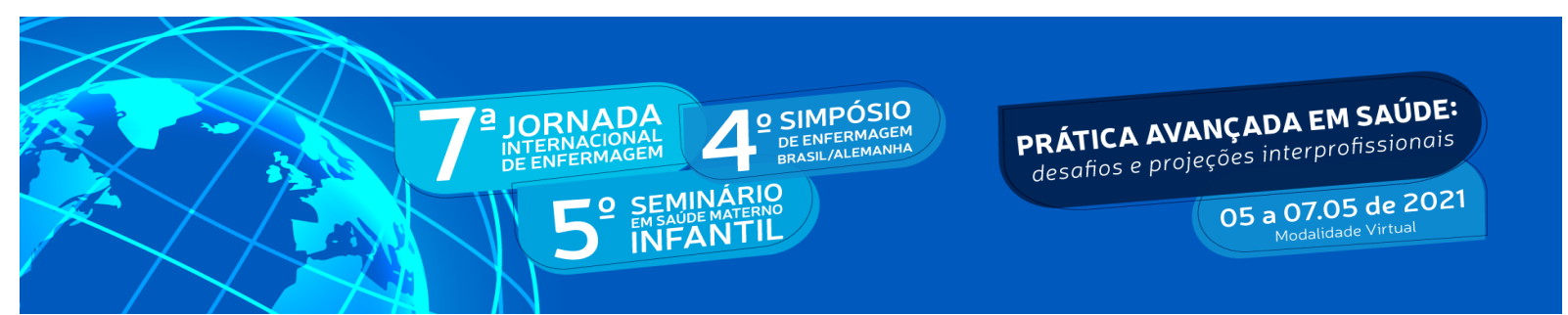

2011; NEGREIROS et al., 2012; RALDI et al., 2019; SARAVANAN et al., 2016). Assim, embora bastante comum, a extração pode ocasionar alguns traumas, sendo necessário um período de recuperação para que os pacientes possam voltar ao estilo de vida habitual após o procedimento (IBIKUNLE; ADEYEMO, 2016; RALDI et al., 2019; SARAVANAN et al., 2016).

O pós-operatório dos pacientes é influenciado por diversos fatores, que podem estar relacionados ao paciente, ao operador e a cirurgia propriamente dita. Alvira-Gonzales et al. (2017) mostraram que vários critérios podem influenciar na dificuldade para remoção dos terceiros molares inclusos e também nas suas complicações. Este estudo identificou 4 variáveis: demográfica, anatômica, radiográfica e operatória. Assim, foi mensurado o grau de relação entre a dificuldade de extração encontrada pela escala analógica visual (EAV) e o tempo de cirurgia. Foi demonstrado que a idade e a posição dentária têm uma correlação positiva com o tempo da operação. Uma idade avançada pode criar dificuldade na cicatrização pós-operatória no nível da densidade óssea, no processo de remodelação (balança osteoblastos/osteoclastos) ou ainda causar certo grau de anquilose. A anatomia do dente também tem um papel importante, sendo que dentes com raízes divergentes normalmente dificultam o procedimento de extração. Os autores também determinaram com uma das complicações mais sérias é o risco de lesar o nervo alveolar inferior, por isso é importante ter em consideração o fator radiográfico em relação ao fator anatômico, para a localização e configuração da impactação dos terceiros molares. Da mesma forma, Lee et al. (2014) revelaram que existe uma correlação positiva entre a dificuldade de extração e as complicações no pós-operatório, sendo que, quanto mais difícil o procedimento cirúrgico, maior a chance de causar infecções do sítio cirúrgico e osteíte alveolar.

Embora nem todo procedimento cirúrgico possa ter complicações pós-operatórias, muitas vezes o uso de medicamentos pode funcionar como reguladores do processo inflamatório/infeccioso. Assim, o uso de antibióticos pode fazer parte dos fármacos de rotina prescritos na cirurgia dos terceiros molares. Existe um grande número de antibióticos disponíveis, tais como a amoxicilina, azitromicina, penicilina $\mathrm{V}$, metronidazol, clindamicina e levofloxacino, além disso, também podemos encontrar combinações, como amoxicilina/ácido clavulânico e amoxicilina/metronidazol. Na literatura não existe um consenso entre os autores para utilização destes fármacos (BRAIMAH et al., 2017; MARCUSSEN et al., 2016; 


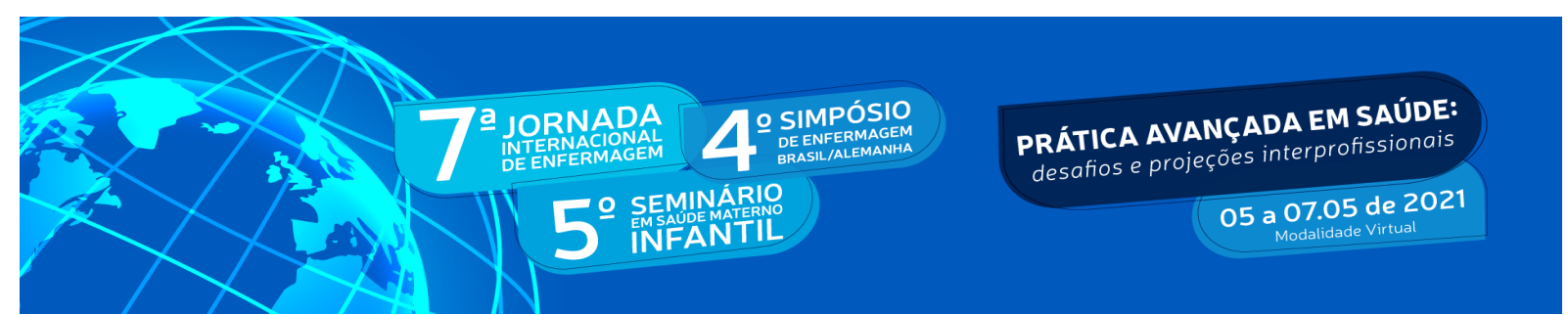

MORROW et al., 2018). Somado a isso, ainda podemos citar que o fármaco pode ser encontrado em diferentes formas, dosagens e vias de administrações. Estes também podem ser administrados de forma profilática (no pré-operatório), somente no pós-operatório, de forma contínua por 5 ou 7 dias, ou ainda de maneira conjunta, no pré e pós-operatório (BLATT; ALNAWAS, 2019; MARCUSSEN et al., 2016; SIDDIQI, MORKEL E ZAFAR, 2010).

Siddiqi, Morkel e Zafar (2010) conduziram um estudo prospectivo randomizado duplamente cego e controlado, com o objetivo de avaliar a eficácia clínica da amoxicilina para prevenção de complicações pós-operatórias em pacientes saudáveis. No estudo, foi realizada a remoção de 380 terceiros inclusos e impactados. Os autores concluíram que a profilaxia antibiótica não se mostrou estatisticamente eficaz para redução de complicações infecciosas e não deve ser administrado rotineiramente nas cirurgias de terceiros molares em pacientes não imunocomprometidos.

Marcussen et al. (2016) realizaram uma revisão sistemática de ensaios clínicos randomizados objetivando encontrar se uma dose única pré-operatória de antibióticos, administrada por via oral e intravenosa, reduziria a incidência e severidade de complicações pós-operatórias, como infecções no sitio cirúrgico (ISC) na cirurgia dos terceiros molares com realização de osteotomia. O estudo concluiu que a dosagem de 2 gramas de amoxicilina no préoperatório reduziu significantemente a incidência de ISC e uma única dose de 0,8 gramas de penicilina V no pré-operatório reduziu a incidência de osteíte alveolar.

Blatt e Al-Nawas (2019) conduziram uma metanálise onde foram analisados ensaios clínicos, estudos retrospectivos, revisões e outras metanálises para verificar se o uso prolongado de antibióticos pode reduzir o risco de ISC. Os autores concluíram que, embora ainda há falta de evidência dos efeitos de curto e longo prazo, a profilaxia com antibióticos mostrou que pode reduzir a ISC, enquanto a dosagem no pós-operatório não mostrou nenhum benefício. Por fim, relataram que nos procedimentos para remoção de terceiros molares a literatura se mostrou ambivalente.

O estado do paciente no pós-operatório pode influenciar diretamente na sua qualidade de vida. Assim, Braimah et al. (2017) examinaram o impacto dos antibióticos orais administrados em dose única no pré-operatório e regime de dose prolongada utilizando levofloxacino e amoxicilina/ácido clavulânico na qualidade de vida relacionada à saúde bucal de pacientes saudáveis. $\mathrm{O}$ autor concluiu que a qualidade de vida após a cirurgia para remoção 


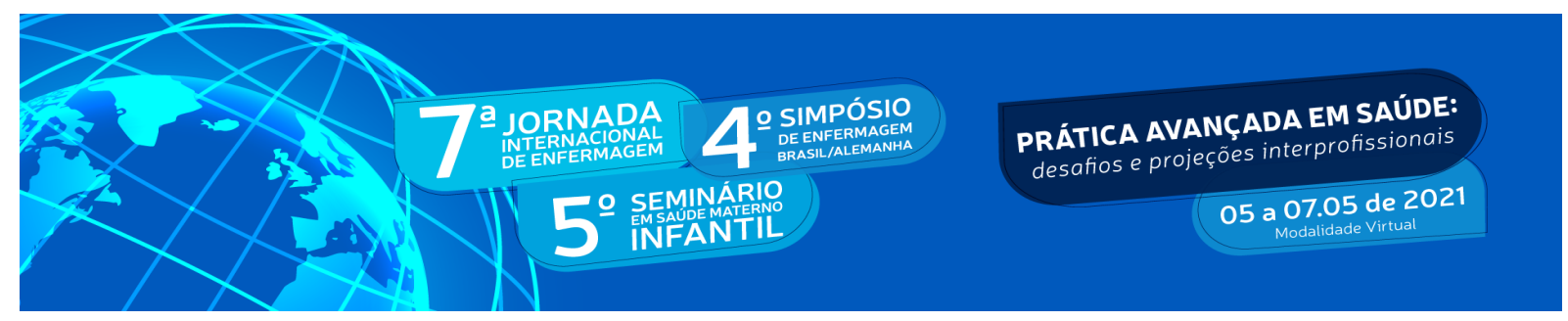

de terceiros molares foi estatisticamente significante e clinicamente relevante nos pacientes que fizeram uso da medicação de amoxicilina/ácido clavulânico 1 grama, 1 hora antes no préoperatório, seguindo de $625 \mathrm{mg} \mathrm{12/12} \mathrm{h} \mathrm{por} 5$ dias quando comparada aos outros grupos que utilizaram amoxicilina 1 grama em dose única no pré-operatório de forma profilática ou ainda aqueles que utilizaram levofloxacino 1 grama também em dose única 1 hora antes do procedimento.

Para comparar a frequência de complicações inflamatórias dos pacientes que removeram os terceiros molares e tiveram o uso de antibióticos apenas no pós-operatório versus aqueles que receberam no pré-operatório, Morrow et al. (2018) realizaram um estudo de coorte em consultórios particulares de 105 cirurgiões bucomaxilofaciais durante um ano. No período, foram extraídos 9123 terceiros molares de 2954 pacientes. O estudo relatou que a osteíte alveolar foi considerada a complicação mais comum no pós-operatório, e teve uma frequência de $4 \%$ nos pacientes que utilizaram antibióticos no pós-operatório e $6,3 \%$ para aqueles que não utilizaram antibiótico. Embora menos comum, a infecção do sítio cirúrgico foi de $0,4 \%$ no pós operatório e 1,4\% para o grupo que não recebeu o medicamento. Embora a cavidade bucal possui um conteúdo bacteriano intrinsicamente alto, muitas precauções pré-cirúrgicas (uso de antibióticos e enxaguatórios bucais) são usadas para reduzir a carga bacteriana que levam a contaminação inerente dos locais cirúrgicos. Dessa forma, os autores concluíram que o uso de antibioticoterapia isolada no pós-operatório independente do padrão de entrega (tipo, dose, frequência e duração) se mostrou eficiente para evitar as complicações inflamatórias após a remoção de terceiros molares.

As infecções pós-operatórias também foram estudo de Brunello et al. (2017). O estudo investigou a ocorrência e as características clínicas das infecções de início tardio na remoção de terceiros molares inferiores. Embora todos os pacientes do estudo fossem medicados no pósoperatório, (clavulanato de amoxicilina $1 \mathrm{~g}$ a cada 12/12 h) e enxaguatório bucal com clorexidina, e mesmo todos os dentistas sendo especialistas e/ou residentes, ainda foram observadas o aparecimento de infecções pós-operatórias. Os autores ainda puderam concluir que, embora raro, este tipo de infecção tem maior frequência em pacientes com menor idade e cujo tempo cirúrgico foi maior, sendo necessário aos cirurgiões informar aos pacientes sobre a possibilidade de infecção mesmo até 4 semanas após a cirurgia. 


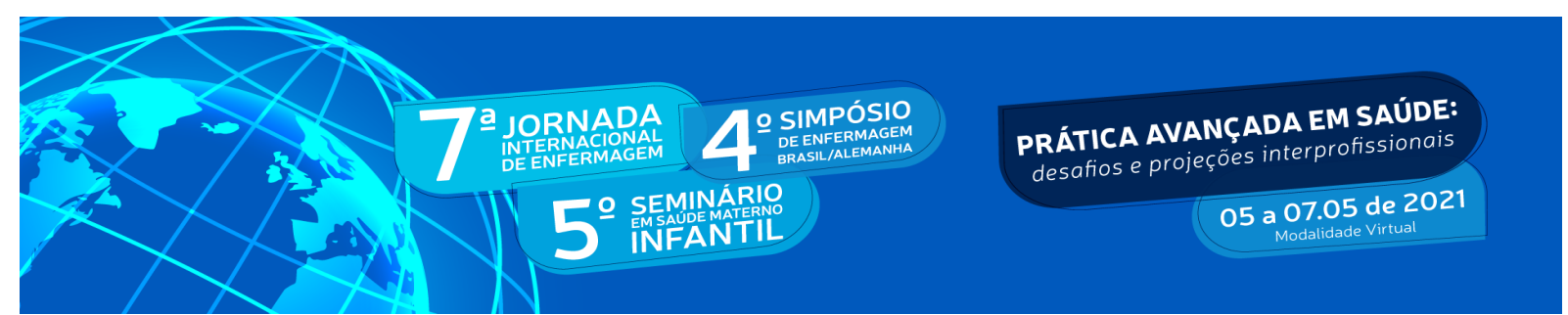

Lodi et al. (2021) realizaram uma revisão sistemática da literatura para determinar o efeito da profilaxia antibiótica sistêmica na prevenção de complicações infecciosas após exodontias de terceiros molares em pacientes saudáveis. O estudo relatou que em comparação com o grupo controle, os antibióticos podem reduzir o risco de complicações infecciosas póscirúrgicas em pacientes submetidos a extrações de terceiros molares em aproximadamente $66 \%$ (RR 0,34, IC de 95\% 0,19 a 0,64; 1728 participantes; 12 estudos; evidência de baixa certeza), o que significa que 19 pessoas (95\% CI 15 a 34) precisam ser tratadas com antibióticos para prevenir uma infecção após a remoção de terceiros molares impactados. Os antibióticos também podem reduzir o risco de alveolite em 34\% (RR 0,66, IC 95\% 0,45 a 0,97; 1882 participantes; 13 estudos; evidência de baixa certeza), o que significa que 46 pessoas (IC 95\% 29 a 62) precisam tomar antibióticos para prevenir um caso de alvéolo seco após a remoção de terceiros molares impactados. A evidência para os outros resultados é incerta: dor, medida dicotomicamente como presença ou ausência (RR 0,59, IC 95\% 0,31-1,12; 675 participantes; 3 estudos) ou continuamente usando uma escala visual analógica ( 0 a 10 centímetros escala, onde 0 é sem dor) (MD -0,26, IC 95\% -0,59 a 0,07; 422 participantes; 4 estudos); febre (RR 0,66, IC 95\% 0,24-1,79; 475 participantes; 4 estudos); e efeitos adversos, que foram leves e transitórios (RR 1,46, IC 95\% 0,81 a 2,64; 1277 participantes; 8 estudos) (evidência de certeza muito baixa). Não foi encontrado também evidências claras de que o momento da administração do antibiótico seja ele no pré-operatório, pós-operatório ou ambos foi importante.

Ao analisar os estudos apresentados percebe que para uma cirurgia seja considerada bem sucedida, ela deve minimizar os desconfortos do paciente no período de pós operatório como as complicações de dor, edema, trismo e também prevenir o desenvolvimento de infecções. O cirurgião deverá estar atento ao correto uso do antibiótico, tanto de forma sistêmica ou de forma profilática quando necessário, evitando assim o aumento da resistência aos antibióticos.

\section{CONSIDERAÇÕES FINAIS}

O uso dos antibióticos podem reduzir complicações infecciosas após a exodontia dos terceiros molares em pacientes não imunocomprometidos, principalmente quando o procedimento cirúrgico para remoção dos terceiros molares envolve osteotomia e ou odontosecção, tempo cirúrgico aumentado ou ainda infecção prévia do sítio cirúrgico, 


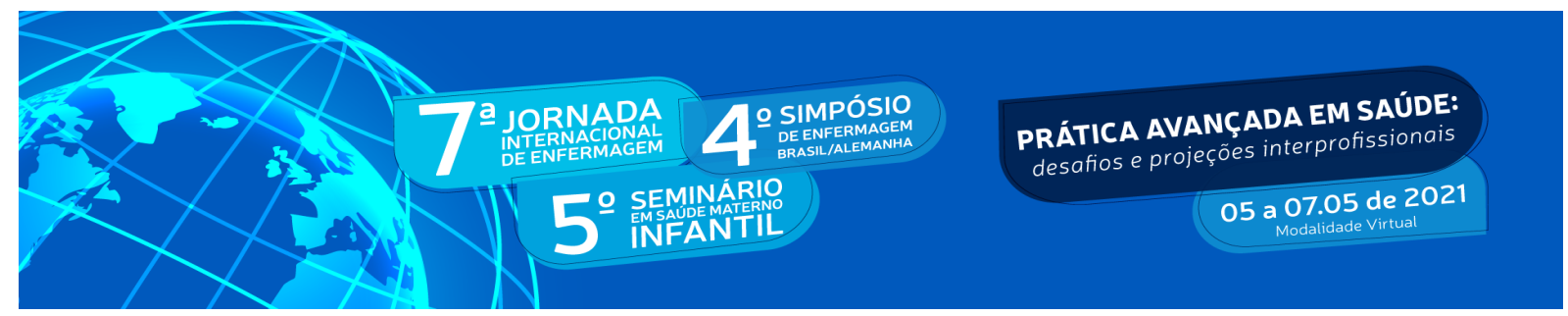

entretanto, o uso da profilaxia antibiótica não se mostrou eficaz para redução das complicações infecciosas, devendo ser utilizado somente em pacientes imunocomprometidos com risco de endocardite bacteriana. Embora os índices de infecções pós operatórios serem baixos, deve-se avaliar bem sua indicação de acordo com o dano causado pelo procedimento cirúrgico.

\section{REFERÊNCIAS}

AL-SHAMIRI, H. M.; SHAWKY, M.; HASSANEIN, N. Comparative assessment of preoperative versus postoperative dexamethasone on postoperative complications following lower third molar surgical extraction. International Journal of Dentistry, Londres, v. 2017, p.1-7, 2017.

ALVIRA-GONZALES, J. et al. Predictive factors of difficulty in lower third molar extraction: a prospective cohort study. Medicina Oral Patologia Oral y Cirurgia Bucal, [s. l.], v. 22, n. 1, p.108-114, 2017.

BLATT, S.; AL-NAWAS, B. A systematic review of latest evidence for antibiotic prophylaxis and therapy in oral and maxillofacial surgery. Infection, [s. l.], v. 47, n. 4, p. 519-555, 2019.

BRAIMAH, R. O. et al. Impact of oral antibiotics on health-related quality of life after mandibular third molar surgery: an observational study. Nigerian Journal of Clinical Practice, Mumbai, v. 20, p. 1189-1194, 2017.

BRUNELLO, G. et al. An observational cohort study on delayed-onset infections after mandibular third-molar extractions. International Journal of Dentistry, Londres, v. 2017, p. $1-5,2017$.

CAYMAZ, M. G.; UYANIK, L. O. Comparison of the effect of advanced platelet-rich fibrin and leukocyte- and platelet-rich fibrin on outcomes after removal of impacted mandibular third molar: a randomized split-mouth study. Nigerian Journal of Clinical Practice, Mumbai, v. 22, n. 4, p. 546-552, 2019.

FERNANDES, I. A.; ARMOND, A. C. V.; FALCI, S. G. M. The effectiveness of the cold therapy (cryotherapy) in the management of inflammatory parameters after removal of mandibular third molars: a meta-analysis. International Archives of Otorhinolaryngology, São Paulo, v. 23, n. 2, p.221-228, 2019.

HAAG, D.G. et al. Oral conditions and health-related quality of life: a systematic review. Journal of Dental Research, [s. l.] v. 96, n. 8, p. 864-874, 2017.

IBIKUNLE, A. A.; ADEYEMO, W. L. Oral health-related quality of life following third molar surgery with or without application of ice pack therapy. Journal of Oral and Maxillofacial Surgery, Nova York, v. 20, n. 3, p. 239-247, 2016. 


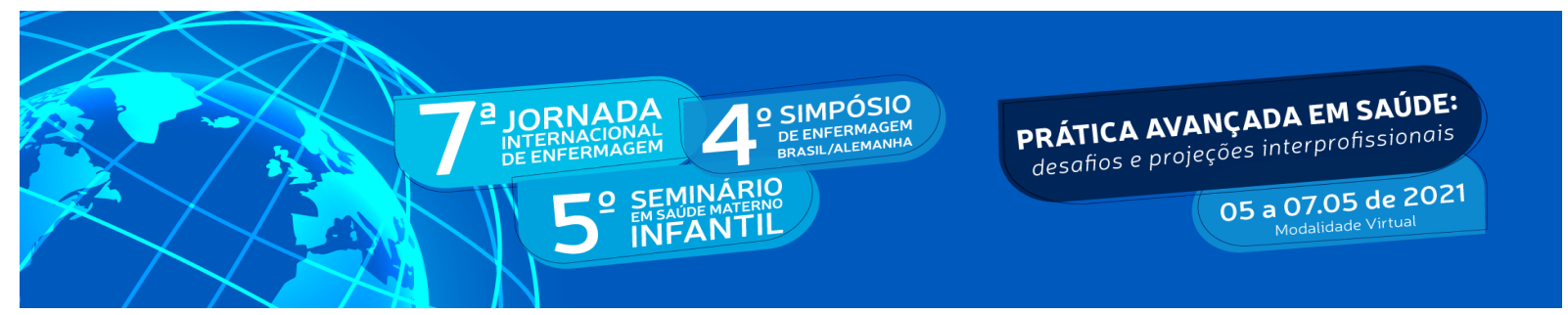

LEE, J. Y. et al. Correlation of antibiotic prophylaxis and difficulty of extraction with postoperative inflammatory complications in the lower third molar surgery. British Journal of Oral and Maxillofacial Surgery, Kidlington, v. 52, n. 1, p. 54-57, 2014.

LODI, G. et al. Antibiotics to prevent complications following tooth extractions. Cochrane Database of Systematic Reviews 2021, Issue 2. Art. No.: CD003811, 2021.

MAHAT, A. K. et al. A comparative study of the effect of sutureless versus multiple sutures technique on complications following third molar surgery in nepalese subpopulation, International Journal of Dentistry, Londres, v. 2020, p. 1-6, 2020.

MAJID, O. W. Submucosal dexamethasone injection improves quality of life measures after third molar surgery: a comparative study. Journal of Oral and Maxillofacial Surgery, Nova York, v. 69, n. 9, p. 2289-2297, 2011.

MARCUSSEN, K. B. et al. A systematic review on effect of single-dose preoperative antibiotics at surgical osteotomy extraction of lower third molars. Journal of Oral and Maxillofacial Surgery, Nova York, v. 74, n. 4, p. 693-703, 2016.

MARKIEWICZ, M. R. et al. Corticosteroids reduce postoperative morbidity after third molar surgery: a systematic review and meta-analysis. Journal of Oral and Maxillofacial Surgery, Nova York, v. 66, n. 9, p.1881-1894, 2008.

MICLOTTE, I. et al. Incidence and treatment of complications in patients whohad third molars orother teeth extracted. British Journal of Oral and Maxillofacial Surgery, Kidlington, v. 56, n. 5, p. 388-393, 2018.

MODANLOO, H.; EFTEKHARIAN, H.; ARABIUN, H. Postoperative pain management after impacted third molar surgery with preoperative oral lamotrigine, a randomized, double-blind, placebo-controlled trial. Journal of Dentistry, Shiraz-Iran, v. 19, n. 3, p. 189-196, 2018.

MORROW, A. J. et al. Do postoperative antibiotics decrease the frequency of inflammatory complications following third molar removal?. Journal of Oral and Maxillofacial Surgery, Nova York, v. 76, n. 4, p. 700-708, 2018.

NEGREIROS, R. M. et al. Relationship between oral health-related quality of life and the position of the lower third molar: postoperative follow-up. Journal of Oral and Maxillofacial Surgery, Nova York, v. 70, n. 4, p.779-786, 2012.

PHAM, T. A. V.; NGUYEN, N. H. Periodontal status of the adjacent second molar after impacted mandibular third molar surgical extraction. Contemporary Clinical Dentistry, Mullana, v. 10, n. 2, p.311-318, 2019. 


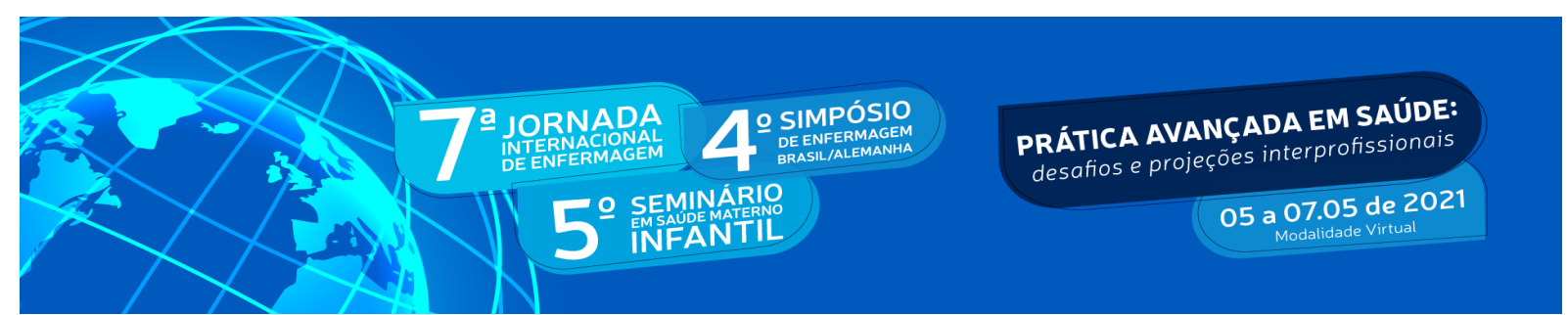

RALDI, F. V. et.al. Evaluation of the impact of preoperative use of dexamethasone and cyclobenzaprine in surgical extraction of lower third molars on trismus by electromyographic analysis. Journal of Oral and Maxillofacial Surgery, Nova York, v. 23, n. 4, p. 395-405, 2019.

RODANANT, P. et al. Pain and quality of life related to suture removal after 3 or 7 days at the extraction sites of impacted lower third molars. Journal of Dental Anesthesia and Pain Medicine, Jongno-gu, v. 16, n. 2, p.131-136, 2016.

SABHLOK, S. et al. Randomized controlled trial to evaluate the efficacy of oral dexamethasone and intramuscular dexamethasone in mandibular third molar surgeries. Journal of Clinical and Diagnostic Research, v. 9, n. 11, p.48-51, 2015.

SARAVANAN, K. et al. A single pre operative dose of sub mucosal dexamethasone is effective in improving post operative quality of life in the surgical management of impacted third molars: a comparative randomised prospective study. Journal of Oral and Maxillofacial Surgery, Nova York, v. 15, n. 1, p. 67-71, 2016. 